\title{
La formation professionnelle des femmes en France
}

\section{Nicole Mosconi}

Les fermmes ont aujourd'hui plus de chances que les hommes de sortir du système scolaire avec une formation générale. Mais la segmentation du marché du travail entre emplois masculins valorisés et emplois féminins dévalorisés se répercute au niveau du système de formation initiale dans une forte ségrégation des garçons et des filles entre filières et cursus différents (littéraires et tertiaires pour les filles, scientifiques et techniques industriels pour les garçons). La poussée de la scolarisation féminine tend à introduire une division verticale dans le groupe des filles entre celles qui ont un niveau égal ou supérieur au baccalauréat et celles qui ont un niveau inférieur. La manière dont s'organisent les différences de formation professionnelle et d'insertion entre les sexes renvoie aux rapports sociaux de sexe qui sont structurés comme un système de division et de domination entre groupes de sexe.

Cet article traitera de la formation professionnelle initiale des femmes et non pas de la formation continue. Celle-ci est une nébuleuse tellement complexe en France qu'elle nécessiterait un article entier à elle seule. Pendant longtemps on s'est peu intéressé à la formation professionnelle des femmes. Les recherches concernant les filières professionnelles dans le système scolaire français concernaient prioritairement - souvent sans le dire - les garçons. On a eu tendance à mettre l'accent sur les progrès de la scolarisation des filles ${ }^{1}$, sans trop se soucier de savoir si cet accroissement considérable des taux de scolarisation assurait aux filles une véritable formation professionnelle et se traduisait par des bénéfices équivalents sur le marché du travail. Il faut attendre 1997 pour que le CEREQ (Centre d'Etudes et de Recherches sur les Qualifications) publie une Étude intitulée Femmes sur le marché du travail. L'autre relation formation-emploi. ${ }^{2}$ Cette étude fait apparaître un des problèmes centraux de la scolarisation des filles aujourd'hui: elles se maintiennent, en plus forte proportion que les garçons et plus longtemps qu'eux, dans les filières générales, par opposition aux filières techniques ou professionnelles, de sorte qu'elles ont plus de chances que les garçons de sortir du système scolaire avec une formation générale, mais sans véritable formation professionnelle. 
D'autre part, la segmentation du marché du travail entre emplois féminins et emplois masculins, les uns dévalorisés et les autres valorisés, se répercute au niveau du système de formation initiale dans une forte ségrégation des garçons et des filles entre filières et cursus différents et inégalement rentables sur le marché du travail.

Pour le montrer, nous étudierons dans une première partie les divisions qui se créent dans le système de formation lui-même entre filières masculines et filières féminines, en particulier dans les filières techniques et professionnelles. Dans une seconde partie, nous montrerons comment la poussée de la scolarisation féminine tend à introduire une division verticale à l'intérieur même du groupe social des filles entre celles qui atteignent un niveau de formation inférieur au baccalauréat et celles qui ont un niveau égal ou supérieur au baccalauréat. Et, dans une dernière partie, nous tenterons une interprétation sociologique de ces phénomènes en termes de rapports sociaux de sexe.

\section{La division du système de formation initiale entre filières masculines et filières féminines}

On pourrait penser qu'aujourd'hui la mixité, impliquant l'unification complète des cursus et des filières, assure un égal accès des deux sexes à toutes les filières de formation. Mais il suffit d'étudier de près les orientations pour découvrir qu'il existe des différences considérables dans la formation des filles et des garçons.

On sait que les filles ont, globalement, une meilleure réussite scolaire que les garçons. Ce fait a pour conséquence que, dans un système scolaire français qui valorise les filières générales au détriment des filières techniques, les filles se maintiennent en plus grand nombre dans l'enseignement général. Mais cet avantage à court terme risque de se transformer en inconvénient dans la mesure où les filles sont moins orientées vers des filières assurant une formation professionnelle.

\section{Lycées professionnels (formations niveau V et IV)}

À l'issue du premier cycle de l'enseignement secondaire, qui est commun à tous les élèves, moins de filles que de garçons sont orientées vers les filières professionnelles de l'enseignement court, les lycées professionnels; elles sont plus souvent orientées vers l'enseignement long, ce qui explique qu'elles représentaient, en 1997, 56\% des lauréats du baccalauréat général et technologique.

Mais surtout, dans ces lycées professionnels, qui préparent BEP (Brevet d'Enseignement Professionnel, niveau V) et Baccalauréat Professionnel (niveau IV), alors que la mixité est la règle de droit, comme dans tout le système scolaire français, celle-ci n'y est pratiquement jamais réalisée. C'est même à ce niveau qu'on observe les plus fortes ségrégations. Les garçons sont massivement dans les filières industrielles et artisanales. Dans le secteur secondaire, on ne trouve de filles 
que dans les sections «matériaux flous» (textiles). Les filles sont surtout présentes dans les filières tertiaires (employées de bureau, de collectivité, secrétariat, santé, services). Notons que l'apprentissage, qui s'est développé ces dernières années, est largement réservé aux garçons, les filles ne représentent que 29,6\% des apprentis. $^{3}$

Au niveau du baccalauréat professionnel ${ }^{4}$, que l'on peut préparer à la suite du $\mathrm{BEP}$, on retrouve la même ségrégation qu'en BEP: les filles représentent $46,4 \%$ des admis, divisés en deux domaines, le domaine de la production où elles représentent $8,2 \%$ et celui des services où elles représentent $71 \%$. Dans les métiers de la production, la seule exception est l'option "productique matériaux souples», où elles sont 95,2\%; dans les autres options industrielles, la proportion oscille entre 0,6 et $5 \%$. Et ces proportions restent stables depuis plus de dix ans, malgré toutes les actions ministérielles pour inciter les filles à s'orienter vers ces sections.

\section{Lycée général et technologique (diplômes niveau IV)}

Dans l'enseignement secondaire long on observe encore de grandes disparités dans l'enseignement technologique ${ }^{5}$, plus professionnalisant, où on retrouve la même dichotomie que dans l'enseignement professionnel entre filières industrielles très majoritairement masculines $(5,7 \%$ de filles au bac STI, Sciences et Technologies Industrielles) et filières tertiaires majoritairement féminines (63,5\% en STT, Sciences et Technologies Tertiaires, avec 89,9\% dans l'option action et communication administrative et plus de $90 \%$ en sciences médico-sociales).

Dans l'enseignement général ${ }^{6}$, si la ségrégation est moins radicale, les filles cependant sont très majoritaires dans la section L (littéraire) $(81,9 \%)$, majoritaires dans la section ES (Économique et Sociale) (61,7\%), et minoritaires dans les sections $S$ (Scientifiques), (43,1\%, avec 41,3 en option mathématiques et $38,1 \%$ en physique-chimie).

D'une manière très schématique, on peut résumer la situation en disant que les filles obtiennent à $70 \%$ un bac littéraire, économique ou tertiaire et les garçons, à 60\% un bac scientifique ou technique industriel. Notons que les garçons sont moins concentrés dans les mêmes sections que les filles, ils ont plus de variété dans leurs choix.

\section{L'enseignement supérieur court (niveau III)}

Dans l'enseignement supérieur court, qui représente deux ou trois années d'études après le baccalauréat et donne une formation professionnelle, les mêmes disparités se retrouvent évidemment que dans les filières de l'enseignement secondaire. Les STS (Sections de Techniciens Supérieurs) et les IUT (Instituts Universitaires de Technologie) se divisent en sections secondaires, masculines, (des sections comme mécanique générale, électricité, électronique sont presqu'exclusivement masculines) et sections tertiaires, plus féminisées (des sections comme matériaux souples, services aux personnes, communication et information sont 
féminines à plus de 75\%). Un autre domaine est très fémininisé (à plus de 90\%): les écoles qui forment les assistant-e-s de services sociaux et les infirmier-ère-s.

\section{L'enseignement supérieur long (niveau II et I)}

À l'université, les filles sont devenues égales en nombre aux garçons, en France, en 1971. Actuellement elles prolongent l'avantage acquis au niveau bac: elles représentent $56,1 \%$ des étudiants $(56,3 \%$ en premier cycle, $58,5 \%$ en second cycle, $49,7 \%$ en troisième cycle, à ce niveau elles rattrapent rapidement les garçons, elles étaient $42 \%$ en 1990).

Mais on retrouve les mêmes disproportions que dans les filières générales de l'enseignement secondaire. En $1997^{7}$, les filles sont très majoritaires en lettres $(74,7 \%)$, en sciences humaines et sociales $(65,2 \%)$, majoritaires en droit $(61,7 \%)$, légèrement majoritaires en sciences de la vie et de la terre $(56,1 \%)$, en médecine $(53,7 \%)$, légèrement minoritaires en sciences économiques et gestion $(47,4 \%)$, et nettement minoritaires en mathématiques et en physique $(35,1 \%)$.

D'autre part, les filles sont très minoritaires dans les sections réputées les plus "prestigieuses» du système scolaire français, les classes préparatoires aux grandes écoles. ${ }^{8} \mathrm{Si}$ les filles représentent $75 \%$ des classes préparatoires littéraires (préparant aux Écoles Normales Supérieures littéraires), 55,3\% des classes économiques et commerciales (préparant aux Écoles Supérieures de Commerce), elles sont $25 \%$ dans les classes préparatoires scientifiques (préparant aux grandes Écoles d'Ingénieurs, dans lesquelles se recrutent les cadres dirigeants de la nation) et elles représentent moins d'un diplômé sur cinq sortant de ces écoles.

On voit que le système français, mixte en théorie dans toutes ses filières, opère en réalité aujourd'hui encore un partage des élèves selon le sexe: filles et garçons ne suivent pas les mêmes filières ni les mêmes cursus de formation, ils et elles n'accèdent pas aux mêmes savoirs ni aux mêmes formations et aux mêmes débouchés professionnels. D'autre part, la discrimination sexuelle se marque par le fait que les filières et les spécialités à dominante féminine ont tendance à être dévalorisées, de même que les emplois auxquels elles donnent accès. Ainsi «à niveau de formation identique, la comparaison des cheminements professionnels des jeunes filles et des jeunes gens se révèle systématiquement défavorable aux premières». ${ }^{\text {? }}$

Toutefois ces inégalités s'atténuent au fur et à mesure que le niveau de formation s'élève. Et les progrès réels faits par les filles des classes privilégiées ou moyennes dans certaines filières universitaires (en lettres, en droit et en économie ou en médecine), tendent à faire penser qu'il existe aujourd'hui un clivage interne à la population féminine. 


\section{Inégalités de formation à l'intérieur de la popoulation féminine}

On sait que le taux d'activité des femmes en France ne cesse de s'accroître depuis 1970. Les femmes représentent aujourd'hui $45 \%$ de la population active. Mais ces actives sont inégalement réparties selon leur niveau de formation. En 1996, dans la tranche d'âge des 25-39 ans, on comptait six actives sur dix parmi les non-diplômées et neuf actives sur dix parmi les diplômées de l'enseignement supérieur. ${ }^{10}$ En effet l'emploi est beaucoup moins défavorable aux diplômées qu'aux femmes faiblement ou pas diplômées. Certains parlent même aujourd'hui d'une «fracture» entre deux populations féminines, l'une qui possède un niveau de formation inférieur au baccalauréat, l'autre qui possède des diplômes égaux ou supérieurs au baccalauréat.

\section{Formation et insertion des femmes diplômées de \\ L'enseignement supérieur}

Dans ce dernier groupe, on constate les effets positifs de l'accroissement du taux de scolarisation des filles et de l'allongement des études. La possession d'un diplôme égal et surtout supérieur au baccalauréat leur ouvre des situations assez favorables d'insertion et d'emploi. Sans oublier que le marché du travail reste fortement ségrégué entre emplois masculins et emplois féminins, on peut remarquer que l'évolution de l'emploi a été favorable aux femmes durant les vingt dernières années. Le développement de l'emploi dans les spécialités tertiaires (emplois administratifs, secrétariat, comptabilité, etc) et dans les catégories des cadres et des professions intermédiaires (enseignantes, infirmières, assistantes sociales etc) permet aux femmes sortant du système de formation avec des diplômes de niveau III, d'entrer sur le marché du travail dans d'assez bonnes conditions.

Les femmes qui sont issues des niveaux les plus élevés de l'appareil de formation (niveau I et II), ont opéré des avancées notables dans les emplois de cadres. Les progressions les plus remarquables concernent des catégories exigeant un niveau d'étude élevé, où les diplômes sont strictement définis et valorisés. Les femmes ont progressé surtout chez les cadres de la fonction publique (de 20,3\% en 1983 à $28,7 \%$ en 1995), chez les professeurs et professions scientifiques (de $44,3 \%$ en 1983 à $52,2 \%$ en 1995 ); elles ont un taux relativement élevé aussi $(36,4 \%)$ dans les professions libérales, médecins et professions paramédicales, professions juridiques, architectes, experts-comptables, etc...

Pour ce qui est des cadres commerciaux et administratifs d'entreprise $(19,8 \%$ de femmes en 1983, 32,9\% en 1995) et des ingénieurs et cadres techniques d'entreprise (qui sont passés de 5,6\% en 1983 à 12,7 en 1995), une bonne partie d'entre eux est formée en France dans les grandes écoles, qui assurent une formation académique et professionnelle de haut niveau. Aujourd'hui les femmes représentent presque 1 diplômée sur 2 dans les écoles de commerce et 1 diplô- 
mée sur 5 dans les écoles d'ingénieurs. C'est à ce niveau que les disparités hommes femmes sont les plus faibles en matière d'insertion et de salaire, même si subsistent de fortes différences ensuite dans les déroulements de carrière, selon le sexe. On peut donc dire que, durant la dernière décennie, «les débouchés professionnels des femmes les plus diplômées s'étendent, du fait de leurs atouts scolaires». ${ }^{11}$

\section{Les handicaps des femmes faiblement diplômées}

À l'opposé, les femmes qui sortent du système scolaire sans diplôme ou avec un diplôme de niveau $\mathrm{V}$ se trouvent aujourd'hui dans une situation très critique pour leur insertion professionnelle. Alors que pour les hommes, il est encore possible de trouver un emploi avec un BEP de spécialités industrielles, la situation est beaucoup plus difficile pour les femmes de même niveau de diplôme. Les emplois d'ouvrières sans qualification dans lesquels elles s'inséraient auparavant sont en voie de disparition. Les secteurs où les femmes étaient nombreuses, textile, habillement, chaussure, sont en crise et cette crise se traduit par une forte diminution des emplois offerts et par un accroissement de la technicité des emplois qui subsistent et qui requièrent des formations acquises dans des filières considérées jusqu'ici comme masculines.

Dans le secteur tertiaire, qui est un autre domaine supposé féminin, puisque aujourd'hui à peu près la moitié des femmes qui travaillent sont employées, on observe des changements défavorables aux jeunes femmes qui ont une formation de niveau V. Les évolutions technologiques changent la nature du travail: aux activités traditionnelles de classement et de frappe se substituent des «activités d'accueil, de communication, de mise en forme de documents, de documentation simple» ${ }^{12}$; et changent aussi les modalités de recrutement: les employeurs recherchent des diplômées plus qualifiées, de niveau IV ou III. De plus, à l'encontre des diplômes masculins de différents niveaux du secteur technique industriel qui correspondent à des niveaux de qualification et d'emploi bien distingués, le flou de la définition des emplois tertiaires de bureau et de leur niveau de qualification rend indéterminée la correspondance entre emplois et diplômes féminins du secteur tertiaire, de sorte que les employeurs peuvent faire jouer la concurrence entre diplômées de niveaux différents: «les titulaires de CAP et BEP sont écartées par les bachelières, elles-mêmes supplantées par les titulaires de BTS». ${ }^{13}$ On comprend que ce système de concurrence joue en défaveur des diplômées de niveau $V$ et aboutisse à des "pratiques de surqualification à l'embauche». ${ }^{14}$

On met aujourd'hui beaucoup l'accent sur les emplois tertiaires de service, dits aussi emplois de proximité (employée de maison, aide aux personnes âgées, garde d'enfants). On y voit un débouché possible pour cette population féminine peu ou pas diplômée. Mais ces emplois ont encore aujourd'hui un statut d'emplois de "seconde zone», dont la qualification est largement sous-estimée, $\mathrm{du}$ fait qu'il s'agit d'emplois qui s'exercent dans la sphère domestique, féminine. Le système de formation n'est pas encore parvenu à organiser une formation qui 
leur confere une réelle professionnalité et qui permette la reconnaissance de la qualification correspondant à ces emplois.

Il faut noter aussi que dans ces catégories, les femmes commencent et parfois poursuivent leur activité professionnelle beaucoup plus souvent que les hommes dans des emplois précaires, emplois à durée déterminée, emplois à temps partiel, contrats liés à des aides publiques et qu'elles sont aussi plus souvent au chômage que les hommes.

Toutes ces données mettent en évidence les liens qui unissent l'appareil de formation et l'appareil de production et montrent que les modalités de passage de l'un à l'autre different plus ou moins pour les femmes et pour les hommes et pour les femmes entre elles. Comment peut-on expliquer ces différences de formation et d'insertion et ces disparités selon le sexe?

\section{Disparité de formation et rapports sociaux de sexe}

Pour comprendre les phénomènes de l'orientation scolaire et de la formation différentielles des garçons et des filles, dans le système de formation et les disparités dans les modalités d'insertion professionnelle des femmes et des hommes, c'est une compréhension d'ensemble de la structure sociale qui est nécessaire. On peut faire l'hypothèse que ces différences en effet sont la résultante des rapports sociaux que le système social a institués entre les sexes.

\section{La théorie conflictualiste des rapports sociaux de sexe} On entend par rapport social ${ }^{15}$ une logique d'organisation du social qui fait système à travers l'ensemble des champs du social, c'est-à-dire qu'il donne une dimension systématique à un ensemble d'éléments articulés entre eux et servant une même logique. Cette logique a une pesanteur historique et elle produit une détermination relative des pratiques. Mais cette logique est aussi productrice du social, donc en perpétuel mouvement. Les rapports sociaux produisent donc simultanément du même (il existe des structures stables) et du différent (il y a du changement).

$\mathrm{Au}$ même titre que les divisions entre classes sociales, les rapports sociaux de sexe constituent une structure fondamentale de la société. Ils représentent une structure transversale qui organise tous les sous-sytèmes sociaux, depuis la famille, l'école, le travail et les autres champs de la réalité sociale. Ces rapports sociaux de sexe sont structurés comme un système de division et de domination entre groupes de sexe, qui a été institué «sur le mode du pouvoir de l'un, le masculin, par rapport à l'autre, le féminin». ${ }^{16}$ Il y a un principe macro-social d'antagonisme et de domination qui construit les individus qui en sont les supports en groupes opposés et hiérarchisés, même si, dans le fonctionnement concret, dans sa dimension micro-sociale, il peut y avoir du jeu et donc certaines marges de liberté. 
On peut interpréter les orientations scolaires, les formations professionnelles et les modalités d'insertion professionnelle comme des processus de mise en oeuvre de ces rapports sociaux de sexe.

\section{Rapports sociaux de sexe, formation et emploi}

Les rapports sociaux de sexe sont d'abord mis en oeuvre dans la socialisation familiale et dans la socialisation scolaire, dont l'orientation scolaire est la résultante. Mais les choix d'orientation que font garçons et filles sont aussi très étroitement liés à une anticipation qu'ils-elles font de la réalité du marché du travail. Or, on a vu qu'aujourd'hui encore, le monde professionnel n'est pas réellement mixte et que les hommes et femmes n'occupent pas les même types d'emplois. Il existe toujours une ségrégation entre emplois masculins et emplois féminins ${ }^{17}$, mais aussi une hiérarchie, entre emplois masculins valorisés et emplois féminins dévalorisés.

Cet ensemble de processus aboutit à ce que j'ai appelé une division sociosexuée des savoirs et du travail. ${ }^{18}$ Tout comme les grandes divisions sociales du travail des sociétés modernes entraînent la nécessité d'une transmission différenciée des savoirs, qui s'opère par la division du système scolaire en cursus et filières différenciés et hiérarchisés, de la même façon, la division socio-sexuée du travail se traduit par une division du système scolaire en filières différenciées et hiérarchisées entre les sexes. Les choix d'orientation des filles sont la conséquence de ces rapports sociaux de sexe. Très schématiquement, on pourrait dire que ces orientations préfigurent certaines des hiérarchies professionnelles les plus classiques entre les sexes (médecin-infirmière, homme cadre-femme assistante ou secrétaire, homme professeur d'université-femme professeur de collège ou de lycée, homme contremaître-femme OS...) qui signent la subordination féminine dans la sphère professionnelle comme dans la sphère familiale.

En même temps cette division socio-sexuée du travail et des savoirs évolue avec le temps. Au modèle bourgeois du XIX ${ }^{\mathrm{e}}$ siècle - aux hommes, les savoirs théoriques et le travail dans les divers champs du domaine public et, aux femmes, les savoirs pratiques et le travail (non reconnu comme tel) dans le champ domestique, privé -, s'est substituée, avec la croissance de la scolarisation, une autre division: aux femmes les savoirs littéraires et relationnels et le travail dans le champ tertiaire, aux hommes les savoirs scientifiques et techniques et le travail dans le champ de la production.

Cette division reste une division hiérarchisée pour deux raisons. L'une tient à la distribution des statuts professionnels et des pouvoirs dans la société. Tout d'abord, dans les fonctions d'exécution, on peut distinguer avec Margaret Maruani et Chantal Nicole ${ }^{19}$, «métiers masculins" (correspondant à des formations et des niveaux de qualification bien définis, plus valorisés et mieux rémunérés) et «emplois féminins»(correspondant à des formations variées, des niveaux de qualification flous et des salaires en général bas). Si on admet que les emplois d'ouvriers sont en général des emplois dont les formations et les qualifications sont 
bien codifiées, alors que les employés représentent des emplois à formations et qualifications floues, on remarquera que, parmi les hommes actifs, $46 \%$ sont ouvriers et $14,3 \%$ employés, en 1996, alors que, parmi les femmes actives, $12,7 \%$ sont ouvrières et $55,1 \%$ employées.

La supériorité masculine se marque aussi par une proportion d'emplois de cadres plus importante pour les hommes (15,9\% en 1996) que pour les femmes $(9,7 \%)^{20}$, les femmes cadres gagnant de surcroît en moyenne $27 \%$ de moins que les hommes de cette catégorie. ${ }^{21}$ Dans les fonctions d'encadrement et de pouvoir, les hommes gardent donc un monopole relatif sur les savoirs qui sont sources des pouvoirs essentiels dans la société (techniques, économiques, politiques, symboliques). Il suffit de se rappeler quelle est leur place dans les formations de «haut niveau» dispensées dans les «grandes écoles» qui leur assurent un monopole - relatif, moins absolu qu'il y a seulement vingt ans - sur les fonctions dirigeantes.

Mais les hommes ne monopolisent pas seulement les savoirs qui assurent les pouvoirs essentiels dans le champ économique et politique, ils tendent aussi à monopoliser le pouvoir symbolique, par la production des nouveaux savoirs et des œuvres de culture. Les femmes restent encore minoritaires dans les institutions chargées de produire les savoirs (enseignement supérieur, 31\%, en $1997^{22}$ et, de plus, elles y occupent très peu les positions qui donnent pouvoir sur les politiques de recherche.

L'autre raison tient à la division socio-sexuée du travail en général. Les femmes, malgré leur présence massive sur le marché du travail, restent assignées au travail domestique et éducatif. Ce que l'on décrit, en effet, généralement comme le problème majeur des femmes mariées d'aujourd'hui - sans qu'il soit apparemment le problème des hommes mariés - à savoir la difficile "conciliation» - conciliation qui cependant doit toujours être "harmonieuse» - entre famille et travail peut être entendu comme un double système d'exploitation: dans la famille, où la division du travail contraint les femmes à un travail domestique et éducatif gratuit, non rémunéré, donc exploité; dans le travail professionnel, où ces charges familiales contraignent les femmes à un travail professionnel dévalué. Les femmes, même si cela ne correspond pas à leur situation réelle, sont considérées par les chefs d'entreprise, comme des mères de famille, attachant autant ou plus d'importance à leur famille qu'à leur activité professionnelle, donc indisponibles pour des fonctions à responsabilités. Même si cette conception est autant un alibi qu'une réalité - puisque $40 \%$ des femmes actives n'ont aucun enfant de moins de 18 ans -, il est vrai cependant que, même si une femme mariée travaille professionnellement, le travail domestique et éducatif, pour sa plus grande part, repose sur elle. En 1995, une enquête de l'INSEE montrait que 66\% des hommes mariés n'apportent aucune contribution significative au travail domestique et éducatif. Ces faits expliquent aussi pourquoi les femmes occupent en moyenne des emplois moins élevés dans la hiérarchie, moins bien rémunérés et comportant moins de perspectives de carrière que les hommes. Les hommes, dégagés du travail domestique et éducatif, restent les principaux pourvoyeurs des 
ressources financières et du statut social du foyer et préservent ainsi leur dominance sur le système familial et, par voie de conséquence, sur le marché du travail.

Il ne faut cependant pas négliger les changements et les évolutions. Entre 1980 et 1995, les femmes, en accroissant leur taux de scolarisation longue, ont opéré, comme on l'a vu, des avancées dans toute une série de professions supposant des formations de haut niveau. Avancées modestes, sans doute, mais hautement symboliques. Il ne faut cependant pas oublier que ces avancées ne bénéficient qu'à une petite minorité de femmes (moins de $10 \%$ de la population féminine) pour qui le diplôme serait un «facteur de desserrement des contraintes qui structurent les arbitrages féminins» (Cocouault et Fournier, p. 95). ${ }^{23}$

À l'inverse, à l'autre bout de l'échelle, on a vu aussi que la situation s'était aggravée pour l'insertion des femmes titulaires d'un diplôme de niveau $\mathrm{V}$ et pour les non-diplômées davantage que pour les hommes dans la même situation. Elles sont beaucoup plus fréquemment au chômage et sont beaucoup plus souvent que les hommes dans des emplois précaires.

Ainsi il y a une combinaison complexe des divisions sociales et des divisions sexuées du travail et des savoirs qui contribuent à cantonner les femmes en général dans une situation de domination sexuée et certaines femmes, plus particulièrement, dans une situation de double domination sociale et sexuée.

Les rapports sociaux de sexe entraînent une lutte permanente entre les sexes en ce qui concerne leur position dans l'espace social. Dans cette lutte, étant donné la hiérarchie entre les territoires, ce sont les femmes qui essaient de gagner des territoires masculins. En plus, c'est exactement ce à quoi les incitent les politiques publiques d'égalité des sexes. Les hommes, au contraire, ne voient aucun intérêt à conquérir les territoires féminins, moins valorisés. Ils sont plutôt occupés à la défense de leurs propres territoires contre les femmes qui, si elles y pénètrent, sont vécues comme cherchant à les envahir. ${ }^{24}$

En conclusion, on peut dire que, pour comprendre la manière dont s'organisent les différences de formation professionnelle et les disparités d'insertion entre les sexes, c'est une compréhension d'ensemble de la structure sociale et des rapports sociaux de sexe qui est nécessaire.

Certes, il y a eu, pour les femmes, des transformations indéniables et considérables. En assurant aux femmes l'accès à l'enseignement secondaire et à l'université, les sociétés occidentales du $\mathrm{XX}^{\mathrm{e}}$ siècle ont permis l'accès des femmes aux savoirs savants, aux formations professionnelles et à l'emploi rémunéré, condition d'indépendance économique. Mais il serait illusoire de croire que ce progrès correspond à une suppression pure et simple de la division socio-sexuée des savoirs, tout comme il serait illusoire de croire que l'on ait assisté à une abolition de la division socio-sexuée du travail. Les territoires différenciés n'ont pas été supprimés, ils ont changé de configuration. Il n'y a donc pas eu suppression mais, pour reprendre une expression de Margaret Maruani et Chantal Nicole (1989), «modernisation» de la division socio-sexuée des savoirs et du travail. 
Quant à ces différences d'orientation et de formation entre garçons et filles, ce sont des sociologues femmes qui, les premières, dans la foulée du mouvement néo-féministe des années 70 , les ont posé comme un problème sociologique, nécessitant des analyses et des théorisations, au même titre que les inégalités sociales. Jusque là les sociologues s'étaient aisément contentés d'explications vaguement naturalistes, en termes de «nature», d' "aptitudes», de «motivations», de "goûts», de «mentalités», voire de «vocations» différents. Les femmes sociologues ont montré que ces «aptitudes» ou ces "goûts» étaient une interprétation réaliste de la réalité actuelle des rapports entre les hommes et les femmes. Ce n'est pas seulement hier que le marché du travail était ségrégué, c'est aujourd'hui, et, de même, ce n'est pas hier que les orientations étaient différenciées, c'est aujourd'hui. Les «idées» ne renvoient pas à une réalité passéiste, elles traduisent la réalité actuelle. En traitant conjointement les idées et la réalité, et surtout en tentant d'en rendre compte à l'aide d'une démarche théorique, les femmes sociologues ont pu faire sortir les femmes et les filles de ce qui était jusque là leur lot dans la théorie (sociologique, psychosociologique ou psychologique): leur invisibilité (Spender 1982).

\section{Notes}

1 En 1900, il y avait 624 étudiantes pour 27’000 étudiants, soit 2,2\%. En 1997, il y avait en France 748000 étudiantes pour 585’000 étudiants soit 56\%. Cf. Baudelot, Ch. \& Establet, R. (1992). Allez les filles!, Paris: Seuil, Avant-Propos.

2 Céreq - DLC, Femmes sur le marché du travail. L'autre relation formation-emploi. Étude n ${ }^{\circ} 70$, novembre 1997.

3 Note d'information, Ministère de l'Éducation Nationale de la Recherche et de la Technologie, Direction de la Programmation et du Développement, nº8.09, mai 1998.

4 Cf. Note d'Information, Ministère de l'Éducation Nationale, de la Recherche et de la Technologie, Direction de la Programmation et du Développement, nº8.03, février 1998.

5 Note d'information n ${ }^{\circ} 98.03$, février 1998.

6 Note d'information $\mathrm{n}^{\circ} 98.03$, février 1998.

7 Note d'information $\mathrm{n}^{\circ} 98.09$, mai 1998.

8 Note d'information $\mathrm{n}^{\circ} 98.19$, juin 1998.

9 Femmes sur le marché du travail. L'autre relation formation-emploi. op. cit., Couppié, Th., «Insertion professionnelle des garçons et des filles: le diplôme réducteur d'inégalités», p.62.

10 Enquête sur l'emploi 1996, INSEE, traitements CEREQ.

11 Cacouault, M., \& Fournier, Ch., Le diplôme contribue-t-il à réduire les différences entre hommes et femmes sur le marché du travail? In Mosconi, N. (Ed.) (1998), Égalité des sexes en éducation et formation. p.82, Paris: PUF.

12 Femmes sur le marché du travail. L'autre relation formation-emploi, op. cit.; "Quelle formation professionnelle pour les jeunes filles?», p.19.

13 Femmes sur le marché du travail. L'autre relation formation-emploi, op. cit.; Pournier. Ch., «Femmes des années 90: diplôme, activité et emploi», p.57.

14 Femmes sur le marché du travail. L'autre relation formation-emploi, op. cit.; Coupié, Th. \& Épiphane, D., «Formation au masculin, insertion au féminin?», p.95. 
15 Cf. Combes, D., Daune-Richard, A.-M. \& Devreux, A.-M. (1991). Mais à quoi sert une épistémologie des rapports sociaux de sexe? In Hurtig M.-C., Kail, M. \& Rouch, R., Sexe et genre. De la hiérarchie entre les sexes. CNRS.

16 Collin, F. (1990). Savoir et différence des sexes. Paris: Les Cahiers du GRIF, 45, p. 84.

17 Rappelons que $76 \%$ des femmes travaillent dans le secteur tertiaire, que 6 groupes d'emplois représentent $60 \%$ de l'emploi féminin: enseignantes, professions intermédiaires de la santé et du travail social, employées civiles, employées administratives d'entreprise, employées de commerce et personnels de services directs aux particuliers $(9,8 \%$ pour les hommes). Sur 255 professions, il existe 167 professions où les femmes sont moins de $10 \%$.

18 Mosconi, N. (1994). Femmes et savoir. La société, l'école et la division sexuelle des savoirs. Paris: L'Harmattan.

19 Maruani, M., Nicole, Ch. (1989). Au labeur des dames. Métiers masculins, emplois féminins. Paris: Syros, Alternatives.

20 Source: INSEE, enquête sur l'emploi 1996.

21 Cf. Les femmes - Contours et caractères, INSEE et Service des droits des femmes, 1995.

22 Note d'information $\mathrm{n}^{\circ} 98.13$, mai 1998.

23 Cacouault, M. \& Fournier, Ch., op. cit., p.95.

24 Cf. Mosconi, N. (Ed.) (1998). Égalité des sexes en éducation et formation. Paris: PUF.

25 Maruani, M. \& Nicole, Ch. (1989). Au labeur des dames. Métiers masculins, emplois féminins. Paris: Syros, Alternatives.

26 Cf. Spender, D. (1982). Invisible women, The schooling scandal. London: Writers and Readers Publishing Cooperative Society Ltd.

\section{Die Berufsbildung der Frauen in Frankreich}

\section{Zusammenfassung}

Die Frauen haben heute in Frankreich die besseren Chancen als die Männer, das Schulsystem mit einem allgemeinbildenden Abschluss zu verlassen. Aber die Segmentierung des Arbeitsmarktes in gesellschaftlich hoch bewertete männliche und gesellschaftlich gering bewertete weibliche Beschäftigungen spiegelt sich im Bildungssystem in einer starken Segregation von Knaben und Mädchen zwischen unterschiedlichen Ausbildungsgängen und -kursen (literarische und auf den Tertiärsektor ausgerichtete für Mädchen, wissenschaftlich und technisch orientierte für Knaben). Die vermehrte Beschulung von Mädchen führte zu einer vertikalen Trennung der Mädchen in zwei Gruppen: Die eine verfügt mindestens über einen Abschluss auf dem Niveau eines Baccalauréats, die andere über eine geringere schulische Qualifikation. Diese Differenzen in der Berufsbildung und die unterschiedliche Einbindung der Geschlechter verweisen auf die gesellschaftlichen Geschlechterbeziehungen, die der Logik von Trennung und Dominanz folgen. 


\section{La formazione professionale delle donne in Francia}

\section{Riassunto}

Attualmente le donne in Francia hanno maggiori possibilità di lasciare il sistema scolastico con una formazione generale. Ma la segmentazione del mercato del lavoro tra impieghi maschili di alto e impieghi femminili di scarso valore si ripercuote sul sistema della formazione iniziale provocando una manifesta separazione tra ragazzi e ragazze in corsi e curricoli diversi (letterari e legati al terziario per le ragazze, scientifici e tecnico-industriali per i ragazzi). La spinta verso una maggiore formazione femminile tende a produrre una divisione verticale tra le ragazze che hanno una formazione equivalente o superiore alla maturità e quelle con una formazione inferiore. L'articolazione delle differenze nella formazione e nell'inserimento professionale sullo sfondo dell'appartenenza sessuale, rinvia così ai rapporti sociali tra i sessi, a loro volta strutturati secondo una logica di potere.

\section{The vocational training of women in France}

\section{Summary}

Women today are more likely than men to emerge from the school system with a general education. However, the segmentation of the labour market between valorised jobs for men an devalued jobs for women is reflected, as far as the system of initial education is concerned, in a significant segregation between boys and girls within different subjects and courses (literary and tertiary for girls, scientific and industrial technology for boys). The growth of feminine education is inclined to introduce a vertical division in the girls' group between those who have a level of education equal to or superior to GCSE «A» Level* and those who have a lower level. The way in which the differences between the sexes are organised in professional training and insertion reflects the social relations between the sexes, which are structured like a system of division and domination between two sexes.

*GCSE «A» Level (anglais) / a High School Diploma (américain) 